\title{
Chemically reactive flow of upper-convected Maxwell fluid with Cattaneo-Christov heat flux model
}

\author{
Muhammad Ijaz Khan ${ }^{1}$ Muhammad Waqas ${ }^{2}$ - Tasawar Hayat ${ }^{1,2}$ • \\ Muhammad Imran Khan ${ }^{3}$ Ahmed Alsaedi ${ }^{2}$
}

Received: 13 February 2017 / Accepted: 8 September 2017/Published online: 25 September 2017

(c) The Author(s) 2017. This article is an open access publication

\begin{abstract}
This attempt concentrates on impact of chemically reactive flow of upper-convected Maxwell liquid. Nonlinear slip condition for Maxwell fluid is employed. The processes of heat and mass transfer through theory of Cattaneo-Christov flux are studied. Ordinary differential systems have been considered. Convergent solutions are constructed for the governing equations. Incoming nonlinear modeled problems have been computed for the velocity, temperature and concentration. The impact of emerging variables, namely Deborah number $(\beta)$, Schmidt number $(S c)$, Thermal relaxation parameter $(\gamma)$, Prandtl number $(P r)$ and chemical reaction parameter $(\delta)$ on quantities of interest is graphically investigated. Both temperature and concentration fields decay when thermal relaxation and chemical reaction parameters are increased.
\end{abstract}

Keywords Upper-convected Maxwell fluid · Chemical reaction $\cdot$ Velocity slip $\cdot$ Non-Fourier flux

Technical Editor: Cezar Negrao.

Muhammad Ijaz Khan

mikhan@math.qau.edu.pk

$\triangle$ Muhammad Imran Khan

mk42@hw.ac.uk

1 Department of Mathematics, Quaid-i-Azam University, 45320, Islamabad 44000, Pakistan

2 Nonlinear Analysis and Applied Mathematics (NAAM) Research Group, Department of Mathematics, Faculty of Science, King Abdulaziz University, P. O. Box 80257, Jeddah 21589, Saudi Arabia

3 Heriot Watt University, Edinburgh Campus, Edinburgh EH14 4AS, UK

\section{List of symbols}

$u, v \quad$ Velocity components

$\lambda_{1} \quad$ Relaxation time material constant

a Positive constant

$D^{*} \quad$ Mass diffusion coefficient

Sc Schmidt number

q Heat flux

$\lambda_{2}$ Relaxation time of heat flux

$T \quad$ Temperature

$k \quad$ Thermal conductivity

$b \quad$ Slip parameter

$T_{\mathrm{w}} \quad$ Temperature of sheet

C Concentration

$C_{\infty} \quad$ Ambient concentration

$C_{\mathrm{w}} \quad$ Concentration of sheet

$\eta \quad$ Dimensionless variable

$\mathbf{L}_{\mathrm{f}} \quad$ Linear operator for velocity

$\mathbf{L}_{\theta} \quad$ Linear operator for temperature

$\mathbf{L}_{\varphi} \quad$ Linear operator for concentration

$T_{\infty} \quad$ Ambient temperature

$\hbar_{\theta} \quad$ Auxiliary parameter for temperature

$\tau \quad$ Cauchy stress tensor

$S \quad$ Extra stress tensor

$\nabla \quad$ Operator

$\rho \quad$ Density

$c_{\mathrm{p}} \quad$ Specific heat

$\alpha \quad$ Thermal diffusivity

$\psi \quad$ Stream function

$\beta \quad$ Deborah number

$\gamma \quad$ Thermal relaxation parameter

$\delta \quad$ Chemical reaction parameter

Pr Prandtl number

$c_{\mathrm{i}} \quad$ Arbitrary constant

$v \quad$ Kinematic viscosity 


$\begin{array}{ll}\frac{\mathrm{D}}{D t} & \text { Convective derivative } \\ K & \text { Reaction rate } \\ \theta & \text { Dimensionless temperature } \\ f_{0} & \text { Initial guess for velocity } \\ \theta_{0} & \text { Initial guess for temperature } \\ \varphi_{0} & \text { Initial guess for concentration } \\ f_{\mathrm{m}}, \theta_{\mathrm{m}}, \varphi_{\mathrm{m}} & \text { General solutions } \\ f_{\mathrm{m}}^{*}, \theta_{\mathrm{m}}^{*}, \varphi_{\mathrm{m}}^{*} & \text { Special solutions } \\ \hbar_{\mathrm{f}} & \text { Auxiliary parameter for velocity } \\ \hbar_{\varphi} & \text { Auxiliary parameter for concentration } \\ \mathbf{j} & \text { Mass flux } \\ \mathbf{L} & \text { Velocity gradient }\end{array}$

\section{Introduction}

There is a wide range of chemical reactions in nature which have widespread practical applications. These reactions are involved in various processes, especially in fog formation and dispersion, food processing, hydrometallurgical industry, air and water pollutions, atmospheric flows, fibers insulation and crops damage due to freezing, etc. In these processes the molecular diffusion of species on the boundary or inside the chemical reaction is very intricate. Some of the reactions have the capacity to proceed gradually or do not react at the moment without catalyst. In this direction Merkin [1] studied a model for isothermal homogeneous-heterogeneous reactions in boundary layer flow over a flat plate. Forced convection stagnation point flow of viscous fluid with homogeneous-heterogeneous reactions was explored by Chaudhary and Merkin [2]. Impact of nanoparticles in flow of viscous liquid with homogeneous/heterogeneous reactions is explored by Krishnamurthy et al. [3]. Khan and Pop [4] put forward such effects on the flow of viscoelastic fluid bounded a stretching sheet. The boundary layer flow of Maxwell fluid over a stretching surface with homogeneous-heterogeneous reactions was examined by Khan et al. [5]. The characteristics of homogeneous-heterogeneous reactions in the region of stagnation point flow of carbon nanotubes towards a stretching cylinder with Newtonian heating were also explored by Hayat et al. [6]. Analysis of homogeneous-heterogeneous reactions in slip flow of Casson liquid towards permeable stretched/shrinked surface is presented by Sheikh and Abbas [7]. Bachok et al. [8] reported heterogeneous-homogeneous reactions in stagnation-point flow towards a stretchable surface. Kameswaran et al. [9] discussed heterogeneous-homogeneous reactions in flow of nanomaterial induced by a permeable stretchable surface. Imtiaz et al. [10] addressed unsteady hydromagnetic flow past a curved stretchable surface subject to heterogeneous-homogeneous reactions. Recently Khan et al. [11] studied heterogeneous-homogeneous reactions in flow of viscous fluid in the presence of viscous dissipation and Joule heating. Hydromagnetic stagnation point flow of viscous liquid toward stretched-shrinked surface with slip condition and heterogeneous-homogeneous reactions is scrutinized by Abbas et al. [12]. Impact of nonlinear thermal radiation and induced magnetic field in flow of viscoelastic material with heterogeneous and homogeneous reactions is reported by Animasaun et al. [13]. Raju et al. [14] examined induced magnetic field effects in flow of Casson liquid with heterogeneous and homogeneous reactions. Qayyum et al. [15] examined heterogeneous-homogeneous reactions flow of silver-water and copper-water nanoparticles in the presence of nonlinear thermal radiation.

It is greatly acknowledged that in circumstances comprising extremely small times, maximal temperatures or thermal gradients nearby absolute zero, heat diffusion concept provided by Fourier becomes imprecise and nonFourier consequence becomes decisive in characterizing the diffusion mechanism and anticipating temperature distribution. Practical prospects where deviance from the Fourier's model turns noteworthy may be encountered, for example, in microelectronic materials including IC chips, heating of laser pulse with high heat flux or exceptionally short duration for hardening of semiconductors, impulse drying and laser surgery in biomedical engineering. Numerous investigations have been reported in order to present new formulation for heat conduction. For instance Straughan [16] disclosed thermal convection phenomenon utilizing non-Fourier heat conduction concept. Non-Fourier heat conduction effectiveness in stretching flow of Maxwell liquid is presented by Han et al. [17]. Waqas et al. [18] extended the idea presented in [17] considering temperature-dependent conductivity. Analysis of radiation and non-Fourier flux in differentially heated two-dimensional square cavity is developed by Sasmal and Mishra [19]. Chemical reaction and stagnation point characteristics in stratified variable thermal conductivity stretched flow of Eyring-Powell material in presence of non-Fourier heat conduction is explored by Hayat et al. [20]. Upper-convected Maxwell liquid flow with non-Fourier heat flux is studied by Saleem et al. [21]. Makinde et al. [22] presented magnetohydrodynamic flow over various geometries with Cattaneo-Christov heat flux.

The aforestated investigations witness that slip effects are not explored correctly. No doubt wall slip appears in complex liquids comprising suspensions, emulsions, foams and polymer analysis. The liquids which communicate boundary slip characteristics possess considerable demands in internal cavities and artificial heart valves [23]. MHD slip flow near a stagnation point in the presence of variable 
thicked surface is investigated by Khan et al. [24] and Babu and Sandeep [25]. Thermal radiation and mixed convection effects in stagnation point slipped flow of viscous liquid is explored by Rashad et al. [26]. Aly and Sayed [27] presented a comparative analysis considering four types of nanoparticles in MHD radiative stretched flow of viscous liquid. Simultaneous influences of mixed convection and magnetohydrodynamics in axisymmetric stretched flow of viscous material through slip effects and convective conditions are analyzed by Ganesh et al. [28]. Hayat et al. [29] established numerical solutions for radiative viscous nanomaterial considering melting effects.

It has been found from the existing information that mostly the flow of non-Newtonian fluid in the presence of slip condition valid for viscous fluid is considered. This is not correct. No doubt the stress in non-Newtonian material even for incompressible case is different than the viscous fluid. Thus, in reality the slip conditions for viscous and non-Newtonian fluids are distinct. Motivated in such fact our prime intention here was to report the slip effects in Maxwell material induced by stretchable surface. This model is capable of predicting relaxation time characteristics [30-34]. Polymer having low molecular weight is the best example for Maxwell material. Note that slip condition in viscous fluid is linear whereas it becomes nonlinear in Maxwell fluid situation. Also the generalized concept of heat and mass fluxes are imposed. Such concept has been used in view of Cattaneo-Christov theory. Another important feature is related to the consideration of stretching surface with chemical reaction. Besides this homotopy concept [35-50] is implemented for arising nonlinear problems. Velocity, temperature and concentration are described for meaningful discussion considering important variables.

\section{Formulation}

Let us consider flow of an incompressible Maxwell fluid over a stretching sheet with slip effect. Heat and mass fluxes have been characterized using Cattaneo-Christov theory. Further, we assume $T_{\mathrm{w}}$ and $C_{\mathrm{w}}$ as the temperature and concentration of stretched surface at $y=0$ while $T_{\infty}$ and $C_{\infty}$ denote the ambient temperature and concentration. The present flow is governed by the following basic expressions:

$\operatorname{div} \mathbf{V}=0$,

$\rho \frac{\mathrm{d} \mathbf{V}}{\mathrm{d} t}=\operatorname{div} \tau$,

$\rho c_{p} \frac{\mathrm{d} T}{\mathrm{~d} t}=\tau . \mathbf{L}-\operatorname{div} \mathbf{q}$, $\frac{\mathrm{d} C}{\mathrm{~d} t}=-\nabla \cdot \mathbf{j}$,

$\mathbf{j}=-D^{*} \nabla C$.

Now (4) gives

$\frac{\mathrm{d} C}{\mathrm{~d} t}=D^{*} \nabla^{2} C$,

in which $C$ represents the concentration of species, $D^{*}$ the mass diffusivity and $\mathbf{j}$ the mass flux.

The Cauchy stress tensor for Maxwell fluid model is

$\tau=-p \mathbf{I}+\mathbf{S}$,

where the extra stress tensor $\mathbf{S}$ satisfies the following relation:

$\mathbf{S}+\lambda_{1} \frac{D \mathbf{S}}{D t}=\mu \mathbf{A}_{1}$,

$\mathbf{A}_{1}=\mathbf{L}+\mathbf{L}^{T}, \mathbf{L}=\nabla \mathbf{V}$.

The velocity, temperature and concentration fields are

$V=[u(x, y), v(x, y), 0], T=T(x, y), C=C(x, y)$.

The boundary layer equations now are

$\frac{\partial u}{\partial x}+\frac{\partial v}{\partial y}=0$,

$u \frac{\partial u}{\partial x}+v \frac{\partial u}{\partial y}=v \frac{\partial^{2} u}{\partial y^{2}}-\lambda_{1}\left(u^{2} \frac{\partial^{2} u}{\partial x^{2}}+v^{2} \frac{\partial^{2} u}{\partial y^{2}}+2 u v \frac{\partial^{2} u}{\partial x \partial y}\right)$,

with

$u-a x+\lambda_{1}\left[u \frac{\partial u}{\partial x}-a u+v \frac{\partial u}{\partial y}\right]=\alpha \frac{\partial u}{\partial y}, v=0 \quad$ at $y=0$,

$u \rightarrow 0$ when $y \rightarrow \infty$.

To study the characteristics of heat and mass transfer we have

$\mathbf{q}+\lambda_{2}\left(\frac{\partial \mathbf{q}}{\partial t}+\mathbf{v} \cdot \nabla \mathbf{q}-\mathbf{q} \cdot \nabla \mathbf{v}+(\nabla \cdot \mathbf{v}) \mathbf{q}\right)=-k \nabla T$.

For $\lambda_{2}=0$ the above equation reduces to Fourier law of heat conduction. For incompressible fluid $\nabla \cdot \mathbf{v}=\mathbf{0}$ and then Eq. (14) gives

$\mathbf{q}+\lambda_{2}\left(\frac{\partial \mathbf{q}}{\partial t}+\mathbf{v} \cdot \nabla \mathbf{q}-\mathbf{q} \cdot \nabla \mathbf{v}\right)=-k \nabla T$.

The energy equation is

$\rho c_{p} \mathbf{v} . \nabla T=-\nabla . \mathbf{q}$.

Equations (15) and (16) yield 


$$
\begin{aligned}
& u \frac{\partial T}{\partial x}+v \frac{\partial T}{\partial y}+\lambda_{2}\left(\begin{array}{c}
u^{2} \frac{\partial^{2} T}{\partial x^{2}}+v^{2} \frac{\partial^{2} T}{\partial y^{2}}+2 u v \frac{\partial^{2} T}{\partial y \partial x}+u \frac{\partial \partial \partial \partial T}{\partial x \partial x} \\
+u \frac{\partial \nu \partial T}{\partial x \partial y}+v \frac{\partial \partial \partial \partial}{\partial y \partial x}+v \frac{\partial \nu \partial \partial}{\partial y \partial y}
\end{array}\right) \\
& \quad=\frac{k}{\rho c_{p}} \frac{\partial^{2} T}{\partial y^{2}},
\end{aligned}
$$

with boundary conditions

$T=T_{w} \quad$ at $y=0, \quad T \rightarrow T_{\infty} \quad$ when $y \rightarrow \infty$

The concentration equation is

$u \frac{\partial C}{\partial x}+v \frac{\partial C}{\partial y}=D^{*} \frac{\partial^{2} C}{\partial y^{2}}-K\left(C-C_{\infty}\right)$,

$C=C_{\mathrm{w}} \quad$ at $y=0, \quad C \rightarrow C_{\infty} \quad$ when $y \rightarrow \infty$.

Employing the transformations

$u=\frac{\partial \psi}{\partial y}, \quad v=-\frac{\partial \psi}{\partial x}, \quad \eta=y \sqrt{\frac{a}{v}}, \quad \psi=x \sqrt{v a} f(\eta)$,

$\theta=\frac{T-T_{\infty}}{T_{\mathrm{w}}-T_{\infty}}, \quad \varphi=\frac{C-C_{\infty}}{C_{\mathrm{w}}-C_{\infty}}$,

the mass conservation law (11) is identically fulfilled and Eqs. $(12,13,17,19,20)$ are reduced to the forms:

$f^{\prime \prime \prime}+f^{\prime 2}+f f^{\prime \prime}+\beta\left(2 f f^{\prime} f^{\prime \prime}-f^{2} f^{\prime \prime \prime}\right)=0$,

$\theta^{\prime \prime}+\operatorname{Pr} f \theta^{\prime}-\operatorname{Pr} \gamma\left(f f^{\prime} \theta^{\prime}+f^{2} \theta^{\prime \prime}\right)=0$,

$\varphi^{\prime \prime}+S c\left(f \varphi^{\prime}-\delta \varphi\right)=0$,

with

$f(\eta)=0, \quad f^{\prime}(\eta)=1-\beta\left[f^{\prime 2}-f^{\prime}\right]+b f^{\prime \prime}(\eta), \quad \theta(\eta)=1$, $\varphi(\eta)=1 \quad$ at $\eta=0$,

$f^{\prime}(\eta) \rightarrow 0, \quad \theta(\eta) \rightarrow 0, \quad \varphi(\eta) \rightarrow 0 \quad$ when $\eta \rightarrow \infty$

The dimensionless variables are

$\beta=\lambda_{1} a, \quad \operatorname{Pr}=\frac{\mu c_{p}}{k}, \quad \gamma=\lambda_{2} a, \quad b=\alpha \sqrt{\frac{a}{v}}$,

$S c=\frac{v}{D^{*}}, \quad \delta=\frac{K}{a}$.

Homotopic solutions and convergence analysis.

We consider $f(\eta), \theta(\eta)$ and $\varphi(\eta)$ via set of base functions

$\left\{\eta^{k} \exp (-n \eta) k / k \geq 0, n \geq 0\right\}$,

in the forms

$f(\eta)=a_{0,0}+\sum_{k=0}^{\infty} \sum_{n=1}^{\infty} a_{k, n} \eta^{k} \exp (-n \eta)$

$\theta(\eta)=\sum_{k=0}^{\infty} \sum_{n=1}^{\infty} b_{k, n} \eta^{k} \exp (-n \eta)$ $\varphi(\eta)=\sum_{k=0}^{\infty} \sum_{n=1}^{\infty} a_{k, n} \eta^{k} \exp (-n \eta)$

where $a_{k, n}, b_{k, n}$ and $c_{k, n}$ are coefficients. Thus all the approximations of $f(\eta), \theta(\eta)$ and $\varphi(\eta)$ must obey the above expressions. The initial approximations and operators are

$$
\begin{aligned}
& f_{0}(\eta)=\frac{1}{1+b}(1-\exp (-\eta)), \\
& \theta_{0}(\eta)=\exp (-\eta), \\
& \varphi_{0}(\eta)=\exp (-\eta), \\
& \mathbf{L}_{f}(f)=\frac{\mathrm{d}^{3} f}{\mathrm{~d} \eta^{3}}-\frac{\mathrm{d} f}{\mathrm{~d} \eta}, \quad \mathbf{L}_{\theta}(\theta)=\frac{\mathrm{d}^{2} \theta}{\mathrm{d} \eta^{2}}-\theta, \\
& \mathbf{L}_{\varphi}(\varphi)=\frac{\mathrm{d}^{2} \varphi}{\mathrm{d} \eta^{2}}-\varphi,
\end{aligned}
$$

with

$L_{\mathrm{f}}\left[C_{1}+C_{2} \exp (\eta)+C_{3} \exp (-\eta)\right]=0$,

$\mathbf{L}_{\theta}\left[C_{4} \exp (\eta)+C_{5} \exp (-\eta)\right]=0$

$\mathbf{L}_{\varphi}\left[C_{6} \exp (\eta)+C_{7} \exp (-\eta)\right]=0$

in which $C_{i}(i=1-7)$ are the arbitrary constants.

The general solutions $\left(f_{\mathrm{m}}, \theta_{\mathrm{m}}\right.$ and $\left.\varphi_{\mathrm{m}}\right)$ in terms of special solutions $\left(f_{m}^{*}, \theta_{m}^{*}\right.$ and $\left.\varphi_{m}^{*}\right)$ are

$f_{\mathrm{m}}(\eta)=f_{\mathrm{m}}^{*}(\eta)+C_{1}+C_{2} \mathrm{e}^{\eta}+C_{3} \mathrm{e}^{-\eta}$,

$\theta_{\mathrm{m}}(\eta)=\theta_{\mathrm{m}}^{*}(\eta)+C_{4} \mathrm{e}^{\eta}+C_{5} \mathrm{e}^{-\eta}$,

$\varphi_{\mathrm{m}}(\eta)=\varphi_{\mathrm{m}}^{*}(\eta)+C_{6} \mathrm{e}^{\eta}+C_{7} \mathrm{e}^{-\eta}$,

where

$C_{2}=C_{4}=C_{6}=0$,

$C_{1}=-\frac{1}{(1+b)}\left(\left.\frac{\partial f_{\mathrm{m}}^{*}(\eta)}{\partial \eta}\right|_{\eta=0}-\left.b \frac{\partial^{2} f_{\mathrm{m}}^{*}(\eta)}{\partial \eta^{2}}\right|_{\eta=0}\right)-f_{\mathrm{m}}^{*}(0)$

$C_{3}=\frac{1}{(1+b)}\left(\left.\frac{\partial f_{\mathrm{m}}^{*}(\eta)}{\partial \eta}\right|_{\eta=0}-\left.b \frac{\partial^{2} f_{\mathrm{m}}^{*}(\eta)}{\partial \eta^{2}}\right|_{\eta=0}\right), \quad C_{5}=-\theta_{\mathrm{m}}^{*}(\eta)$,

$C_{7}=-\varphi_{\mathrm{m}}^{*}(\eta)$.

The convergence of series solutions in HAM procedure is quite necessary. Such convergence analysis heavily depends upon the auxiliary variables $\hbar$. Thus $\hbar$ - curves for $f, \theta$ and $\varphi$ have been displayed in Fig. 1. Figure clearly depicts that the acceptable ranges for values of $\hbar_{\mathrm{f}}, \hbar_{\theta}$ and $\hbar_{\varphi} \quad$ are $\quad\left(-0.7 \leq \hbar_{\mathrm{f}} \leq-0.4,-1.7 \leq \hbar_{\theta} \leq-0.4\right.$ and $\left.-1.9 \leq \hbar_{\varphi} \leq-0.8\right)$. 


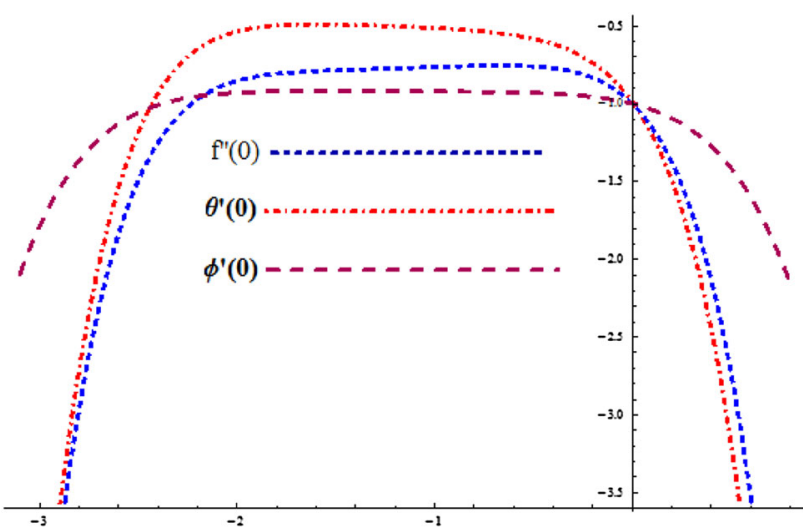

Fig. 1 The $\hbar$-curves for $f^{\prime \prime}(0), \theta^{\prime}(0)$ and $\varphi^{\prime}(0)$ when $\gamma=0.2, \beta=$ $0.3, \operatorname{Pr}=1.2, \delta=1, b=0.1$ and $\mathrm{Sc}=1.2$

\section{Discussion}

This portion presents the impacts of various pertinent parameters like Deborah number $(\beta)$, thermal relaxation parameter $(\gamma)$, chemical reaction parameter $(\delta)$, Prandtl number $(\mathrm{Pr})$, and Schmidt number $(S c)$ on the non-dimensional velocity $f^{\prime}(\eta)$, temperature $\theta(\eta)$ and concentration $\varphi(\eta)$. Rheological behavior of Deborah number $(\beta)$ on the velocity $f^{\prime}(\eta)$ is presented in Fig. 2. It is observed that velocity $f^{\prime}(\eta)$ reduces with enhancement of Deborah number $(\beta)$. From a physical point of view when shear stress is eliminated fluid will come to rest. This sort of phenomenon is shown in many polymeric liquids that cannot be defined in the viscous fluid model. Higher estimation of Deborah number $(\beta)$ will produce a

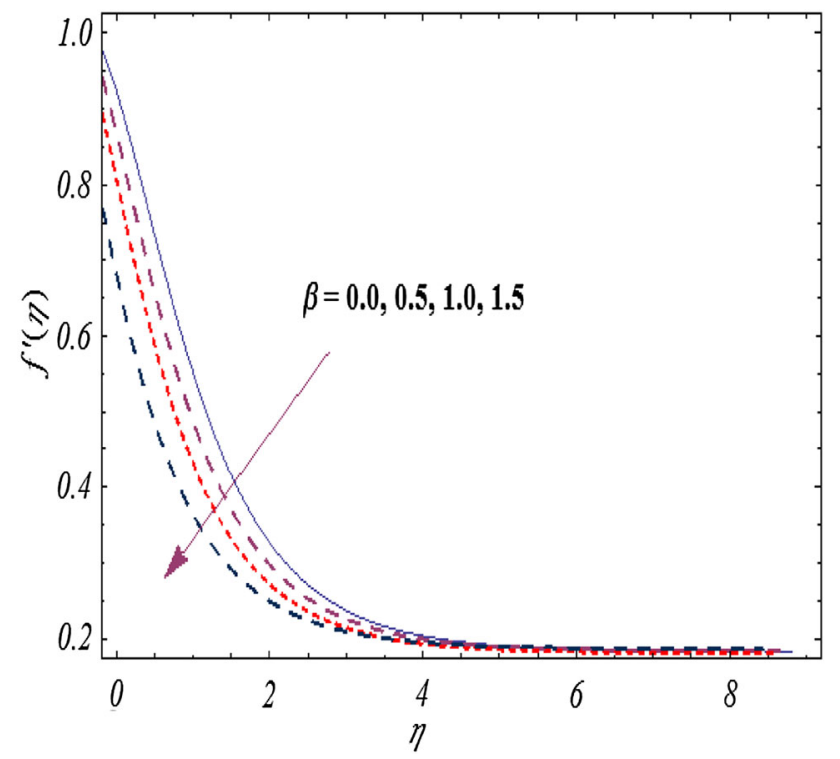

Fig. 2 Variation of $\beta$ for $f^{\prime}$ retarding force between two adjacent layers in the flow. Due to this fact there is a reduction in the velocity and associated layer thickness. The results of viscous fluid are obtained when $\beta=0$. Figure 3 is sketched for the effect of $(\gamma)$ on temperature $\theta(\eta)$. It is scrutinized that temperature $\theta(\eta)$ shows recessive behavior for higher estimation of thermal relaxation parameter $(\gamma)$. It is found that larger values of $\gamma$ decreases both temperature field and thermal layer thickness. Physically for higher estimation of thermal relaxation parameter $(\gamma)$ a material requires more time to transfer heat from more energetic particles to low energetic particles, i.e., it demonstrates the features of a non-conducting material. Therefore, temperature $\theta(\eta)$ decays. Further, it is noted that for $(\gamma=0)$ the heat transfers without any delay through the whole material. Thus temperature $\theta(\eta)$ dominants for Fourier law (i.e., for $\gamma=0$ ) in comparison to CattaneoChristov heat flux model. Behavior of Prandtl number $(\operatorname{Pr})$ on temperature $\theta(\eta)$ is examined in Fig. 4. Higher estimation of $P r$ decays the temperature field and thermal layer thickness. It is due the fact that Pr is the combination of thermal diffusivity to momentum diffusivity. Therefore, small values of $\operatorname{Pr},(P r \ll 1)$ mean the thermal diffusivity dominates. For higher estimation of $\operatorname{Pr}, \quad(\operatorname{Pr} \gg 1)$ the momentum diffusivity dominates. Brownian diffusion coefficient decreases due to which concentration boundary layer is reduced. Influence of chemical reaction parameter $(\delta)$ on concentration $\varphi(\eta)$ is displayed in Fig. 5. As anticipated, a reduction in concentration $\varphi(\eta)$ is observed when the chemical reaction parameter $(\delta>0)$ is increased. Figure 6 illustrates the behavior of Schmidt number $(S c)$ on concentration $\varphi(\eta)$.

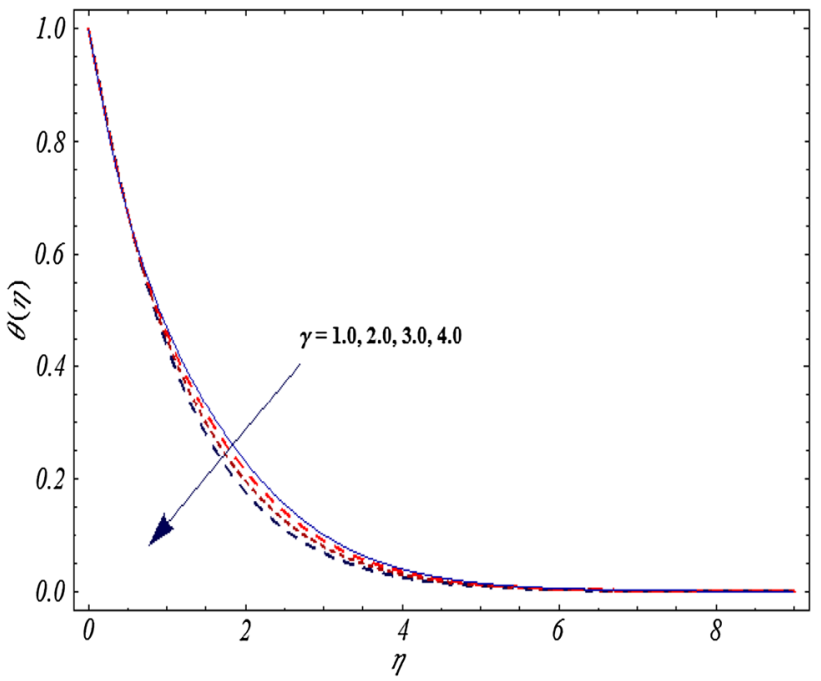

Fig. 3 Variation of $\gamma$ for $\theta$ 


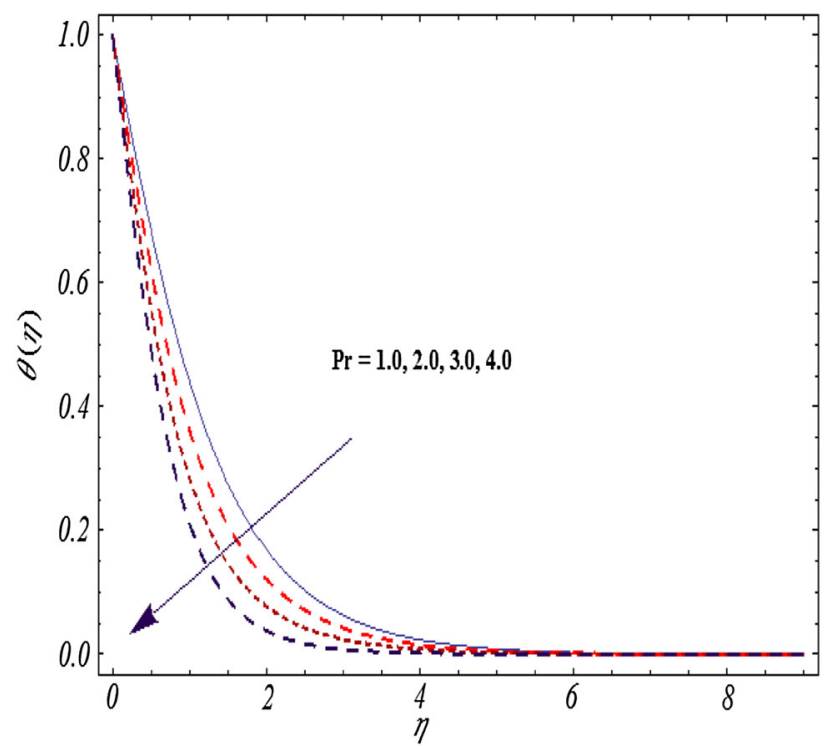

Fig. 4 Variation of $\operatorname{Pr}$ for $\theta$

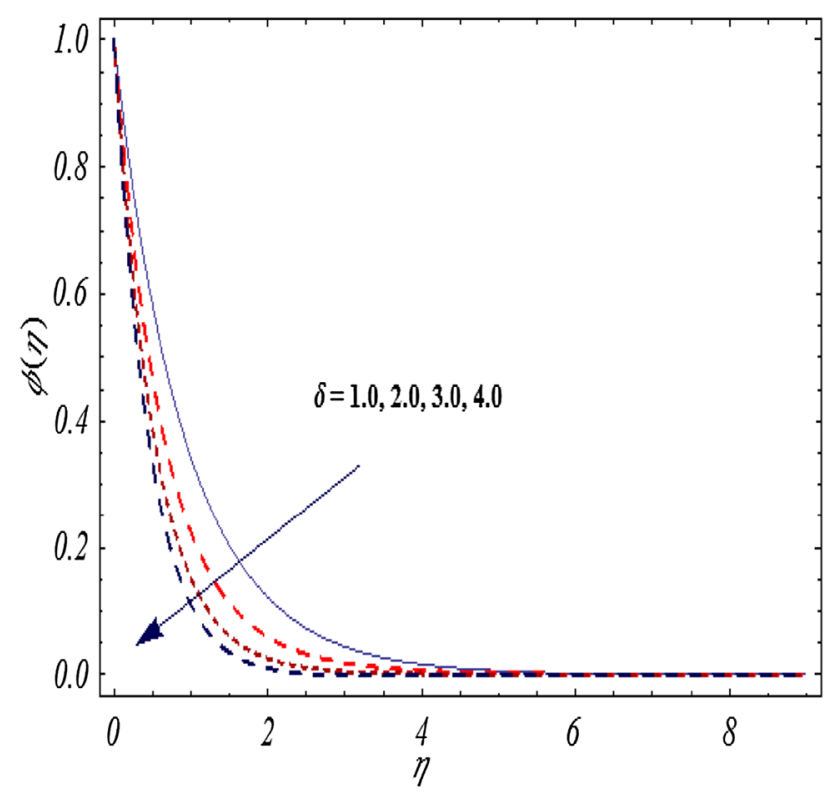

Fig. 5 Variation of $\delta$ for $\varphi$

Ratio of viscous to molecular diffusion rate is known as Schmidt number. Larger viscous diffusion rate is observed when Schmidt number is increased and consequently fluid concentration enhances. Table 1 is constructed to show the order of convergence. $f^{\prime \prime}(0), \theta^{\prime}(0)$ and $\varphi^{\prime}(0)$ converge at 15 th and 16th order of approximations, respectively. To examine the correctness of flow problem, we have compared our results with the published results of Makinde et al. [51] in Table 2. The results are found in an excellent agreement.

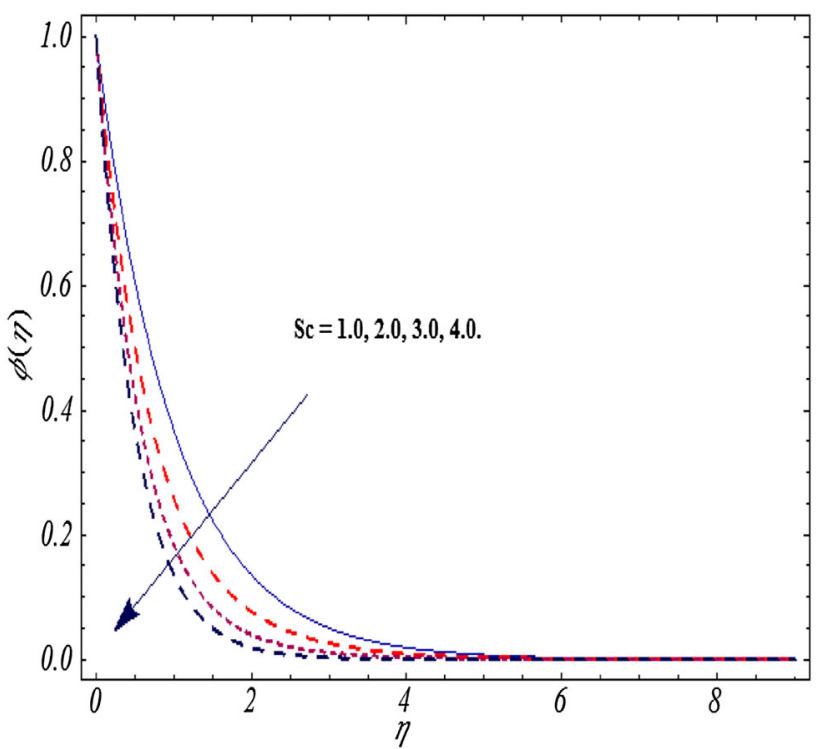

Fig. 6 Variation of $S c$ for $\varphi$

Table 1 Series solutions convergence when $\gamma=0.2, \beta=0.3, \operatorname{Pr}=$ $1.2, \delta=1, b=0.1, h=-1.2$ and $S c=1.2$

\begin{tabular}{llll}
\hline Order of approximation & $-f^{\prime \prime}(0)$ & $-\theta^{\prime}(0)$ & $-\varphi^{\prime}(0)$ \\
\hline 1 & 1.2077 & 0.1599 & 0.04985 \\
3 & 1.2376 & 0.1553 & 0.04970 \\
8 & 1.2427 & 0.1529 & 0.04954 \\
10 & 1.2431 & 0.1527 & 0.04951 \\
15 & 1.2435 & 0.1525 & 0.04945 \\
16 & 1.2436 & 0.1525 & 0.04944 \\
20 & 1.2436 & 0.1525 & 0.04944 \\
24 & 1.2436 & 0.1525 & 0.04944 \\
\hline
\end{tabular}

Table 2 Comparative analysis of present results with [51] for distinct values of $\operatorname{Pr}$ when $\gamma=0$

\begin{tabular}{lll}
\hline & $-\theta^{\prime}(0)$ & $-\theta^{\prime}(0)$ \\
\hline$P r$ & Makinde et al. [51] & Present \\
0.2 & 0.61913 & 0.61913 \\
0.7 & 0.45395 & 0.45395 \\
2.0 & 0.91132 & 0.91132 \\
\hline
\end{tabular}

\section{Closing remarks}

In this paper we employ the upper-convected Maxwell model and non-Fourier heat flux model to investigate heat and mass transfer above a stretching plate with velocity slip. The numerical results suggest the following:

- Velocity decays for higher estimation of $\beta$.

- Temperature field decreases for larger $\operatorname{Pr}$ and $\gamma$. 
- Both $\theta$ and $\varphi$ decay by increasing $\gamma$ and $\delta$.

- Concentration of fluid decreases for higher estimation of $S c$.

Open Access This article is distributed under the terms of the Creative Commons Attribution 4.0 International License (http://crea tivecommons.org/licenses/by/4.0/), which permits unrestricted use, distribution, and reproduction in any medium, provided you give appropriate credit to the original author(s) and the source, provide a link to the Creative Commons license, and indicate if changes were made.

\section{References}

1. Merkin JH (1996) A model for isothermal homogeneousheterogeneous reactions in boundary layer flow. Math Comput Model 24:125-136

2. Chaudhary MA, Merkin JH (1995) A simple isothermal model for homogeneous-heterogeneous reactions in boundary layer flow: I. Equal diffusivities. Fluid Dyn Res 16:311-333

3. Krishnamurthy MR, Prasannakumara BC, Gireesha BJ, Gorla RSR (2016) Effect of chemical reaction on MHD boundary layer flow and melting heat transfer of Williamson nanofluid in porous medium. Eng Sci Technol Int J 19:53-61

4. Khan WA, Pop IM (2012) Effects of homogeneous-heterogeneous reactions on the viscoelastic fluid toward a stretching sheet. J Heat Transf 134:064506

5. Khan MI, Hayat T, Waqas M, Khan MI, Alsaedi A (2017) Impact of heat generation/absorption and homogeneous-heterogeneous reactions on flow of Maxwell fluid. J Mol Liq 233:465-470

6. Hayat T, Farooq M, Alsaedi A (2015) Homogeneous-heterogeneous reactions in the stagnation point flow of carbon nanotubes with Newtonian heating. AIP Adv 5:027130

7. Sheikh M, Abbas Z (2017) Homogeneous-heterogeneous reactions in stagnation point flow of Casson fluid due to a stretching/ shrinking sheet with uniform suction and slip effects. Ain Sham Eng J 8:467-474

8. Bachok N, Ishak A, Pop I (2011) On the stagnation-point flow towards a stretching sheet with homogeneous-heterogeneous reactions effects. Commun Nonlinear Sci Numer Simul 16:4296-4302

9. Kameswaran PK, Shaw S, Sibanda P, Murthy PVSN (2013) Homogeneousheterogeneous reactions in a nanofluid flow due to porous stretching sheet. Int J Heat Mass Transf 57:465-472

10. Imtiaz M, Hayat T, Alsaedi A, Hobiny A (2016) Homogeneousheterogeneous reactions in MHD flow due to an unsteady curved stretching surface. J Mol Liq 221:245-253

11. Khan MI, Hayat T, Khan MI, Alsaedi A (2017) A modified homogeneous-heterogeneous reactions for MHD stagnation flow with viscous dissipation and Joule heating. Int J Heat Mass Transf 113:310-317

12. Abbas Z, Sheikh M, Pop I (2015) Stagnation-point flow of a hydromagnetic viscous fluid over stretching/shrinking sheet with generalized slip condition in the presence of homogeneousheterogeneous reactions. J Taiwan Inst Chem Eng 55:69-75

13. Animasaun IL, Raju CSK, Sandeep N (2016) Unequal diffusivities case of homogeneous-heterogeneous reactions within viscoelastic fluid flow in the presence of induced magnetic-field and nonlinear thermal radiation. Alex Eng J 55:1595-1606

14. Raju CSK, Sandeep N, Saleem S (2016) Effects of induced magnetic field and homogeneous-heterogeneous reactions on stagnation flow of a Casson fluid. Eng Sci Technol Int J 19:875-887
15. Qayyum S, Khan MI, Hayat T, Alsaedi A (2017) A framework for nonlinear thermal radiation and homogeneous-heterogeneous reactions flow based on silver-water and copper-water nanoparticles: a numerical model for probable error. Results Phys 7:1907-1914

16. Straughan B (2010) Thermal convection with the CattaneoChristov model. Int J Heat Mass Transf 53:95-98

17. Han S, Zheng L, Li C, Zhang X (2014) Coupled flow and heat transfer in viscoelastic fluid with Cattaneo-Christov heat flux model. Appl Math Lett 38:87-93

18. Waqas M, Hayat T, Farooq M, Shehzad SA, Alsaedi A (2016) Cattaneo-Christov heat flux model for flow of variable thermal conductivity generalized Burgers fluid. J Mol Liq 220:642-648

19. Sasmal A, Mishra SC (2014) Analysis of non-Fourier conduction and radiation in a differentially heated 2-D square cavity. Int $\mathrm{J}$ Heat Mass Transf 79:116-125

20. Hayat T, Zubair M, Waqas M, Alsaedi A, Ayub M (2017) On doubly stratified chemically reactive flow of Powell-Eyring liquid subject to non-Fourier heat flux theory. Res Phys. doi:10. 1016/j.rinp.2016.12.003

21. Saleem S, Awais M, Nadeem S, Sandeep N, Mustafa T (2017) Theoretical analysis of upper-convected Maxwell fluid flow with Cattaneo-Christov heat flux model. Chin J Phys. doi:10.1016/j. cjph.2017.04.005

22. Makinde OD, Sandeep N, Animasaun IL, Tshehla MS (2017) Numerical exploration of Cattaneo-Christov heat flux and mass transfer in magnetohydrodynamic flow over various geometries. Defect Differ Forum 374:67-82

23. Bhatti MM, Abbas MA, Rashidi MM (2016) Combine effects of Magnetohydrodynamics (MHD) and partial slip on peristaltic Blood flow of Ree-Eyring fluid with wall properties. Eng Sci Technol Int J 19:1497-1502

24. Khan MWA, Waqas M, Khan MI, Alsaedi A, Hayat T (2017) MHD stagnation point flow accounting variable thickness and slip conditions. Colloid Polymer Sci 295:1201-1209

25. Babu MJ, Sandeep N (2016) 3D MHD slip flow of a nanofluid over a slendering stretching sheet with thermophoresis and Brownian motion effects. J Mol Liq 222:1003-1009

26. Rashad AM (2017) Impact of thermal radiation on MHD slip flow of a ferrofluid over a non-isothermal wedge. J Magn Magn Mater 422:25-31

27. Aly EH, Sayed HM (2017) Magnetohydrodynamic and thermal radiation effects on the boundary-layer flow due to a moving extensible surface with the velocity slip model: a comparative study of four nanofluids. J Magn Magn Mater 422:440-451

28. Ganesh NV, Ganga B, Hakeem AKA, Saranya S, Kalaivanan R (2016) Hydromagnetic axisymmetric slip flow along a vertical stretching cylinder with convective boundary condition. St. Petersburg Polytech Univ J Phys Math. doi:10.1016/j.spjpm. 2016.09.001

29. Hayat T, Khan MI, Waqas M, Alsaedi A, Farooq M (2017) Numerical simulation for melting heat transfer and radiation effects in stagnation point flow of carbon-water nanofluid. Computer Methods Appl Mech Eng. doi:10.1016/j.cma.2016.11. 033

30. Han S, Zheng L, Li C, Zhang X (2014) Coupled flow and heat transfer in viscoelastic fluid with Cattaneo-Christov heat flux model. Appl Math Lett 38:87-93

31. Gireesha BJ, Mahanthesh B, Gorla RSR, Krupalakshmi KL (2016) Mixed convection two-phase flow of Maxwell fluid under the influence of non-linear thermal radiation, non-uniform heat source/sink and fluid-particle suspension. Ain Shams Eng J. doi:10.1016/j.asej.2016.04.020

32. Hayat T, Khan MI, Farooq M, Yasmeen T, Alsaedi A (2016) Stagnation point flow with Cattaneo-Christov heat flux and homogeneous-heterogeneous reactions. J Mol Liq 220:49-55 
33. Ibrahim W (2016) The effect of induced magnetic field and convective boundary condition on MHD stagnation point flow and heat transfer of upper-convected Maxwell fluid in the presence of nanoparticle past a stretching sheet. Propul Power Res 5:164-175

34. Hayat T, Qayyum S, Waqas M, Alsaedi A (2016) Thermally radiative stagnation point flow of Maxwell nanofluid due to unsteady convectively heated stretched surface. J Mol Liq 224:801-810

35. Liao SJ (2012) Homotopic analysis method in nonlinear differential equations. Springer, Heidelberg

36. Hayat T, Qayyum S, Imtiaz M, Alsaedi A (2016) Impact of Cattaneo-Christov heat flux in Jeffrey fluid flow with homogeneous-heterogeneous reactions. PLoS One 11:e0148662

37. Hayat T, Qayyum S, Imtiaz M, Alsaedi A (2016) Three-dimensional rotating flow of Jeffrey fluid for Cattaneo-Christov heat flux model. AIP Adv 6:025012

38. Animasaun IL, Adebile EA, Fagbade AI (2016) Casson fluid flow with variable thermo-physical property along exponentially stretching sheet with suction and exponentially decaying internal heat generation using the homotopy analysis method. J Nigerian Math Soc 35:1-17

39. Daniel YS, Daniel SK (2015) Effects of buoyancy and thermal radiation on MHD flow over a stretching porous sheet using homotopy analysis method. Alex Eng J 54:705-712

40. Waqas M, Khan MI, Farooq M, Alsaedi A, Hayat H, Yasmeen T (2016) Magnetohydrodynamic (MHD) mixed convection flow of micropolar liquid due to nonlinear stretched sheet with convective condition. Int J Heat Mass Transf 102:766-772

41. Hayat T, Waqas M, Khan MI, Alsaedi A (2017) Impacts of constructive and destructive chemical reactions in magnetohydrodynamic (MHD) flow of Jeffrey liquid due to nonlinear radially stretched surface. J Mol Liq 225:302-310
42. Shagaiy Y, Aziz ZA, Ismail Z, Salah F (2017) Effects of thermal radiation, viscous and Joule heating on electrical MHD Nanofluid with double stratification. Chin J Phys 55:630-651

43. Daniel YS (2016) Laminar convective boundary layer slip flow over a flat plate using homotopy analysis method. J Inst Eng Ser E 97:115-121

44. Khan MI, Waqas M, Hayat T, Alsaedi A (2017) A comparative study of Casson fluid with homogeneous-heterogeneous reactions. J Colloid Interface Sci 498:85-90

45. Hayat T, Khan MI, Waqas M, Alsaedi A (2017) Newtonian heating effect in nanofluid flow by a permeable cylinder. Res Phys 7:256-262

46. Khan MI, Hayat T, Waqas M, Alsaedi A (2017) Outcome for chemically reactive aspect in flow of tangent hyperbolic material. J Mol Liq 230:143-151

47. Shehzad SA, Hayat T, Alsaedi A, Ahmad B (2015) Effects of thermophoresis and thermal radiation in mixed convection threedimensional flow of Jeffrey fluid. Appl Math Mech 36:655-668

48. Hayat T, Khan MI, Waqas M, Khan MI, Alsaedi A (2016) Impact of Cattaneo-Christov heat flux model in flow of variable thermal conductivity fluid over a variable thicked surface. Int J Heat Mass Transf 99:702-710

49. Hayat T, Khan MI, Waqas M, Alsaedi A, Khan MI (2017) Radiative flow of micropolar nanofluid accounting thermophoresis and Brownian moment. Int $\mathbf{J}$ Hydrol Energy 42:16821-16833

50. Khan MI, Waqas M, Hayat T, Alsaedi A (2017) Magnetohydrodynamic (MHD) stagnation point flow of Casson fluid over a stretched surface with homogeneous-heterogeneous reactions. J Theor Comput Chem 16:1750022

51. Makinde OD, Aziz A (2011) Boundary layer flow of nanofluid past a stretching sheet with a convective boundary condition. Int J Therm Sci 50:1326-1332 